\title{
Asymmetric Synthesis of Methyl (-)-13-Oxo-15,16-dinorlabda-8(17),11E- dien-19-oate, Methyl Ester of a Potent Suppressor toward Carcinogenic Promotor
}

\author{
Takahiro Katoh, Shinsuke Mizumoto, Masato Fudesaka, Tetsuya Kajimoto, and Manabu Node* \\ Department of Pharmaceutical Manufacturing Chemistry, 21st Century COE Program, Kyoto Pharmaceutical University; \\ 1 Shichono-cho, Misasagi, Yamashina-ku, Kyoto 607-8412, Japan. \\ Received May 11, 2006; accepted June 9, 2006; published online June 13, 2006
}

Asymmetric synthesis of methyl ester (4) of (-)-13-oxo-15,16-dinorlabda-8(17),11E-dien-19-oic acid (1), which exhibited the most potent activity for the prevention of incipient carcinogenesis among the isolated diterpenes from Thuja standishii and its related plants, was achieved by using methyl (-)-1,4a-dimethyl-5-oxodecahydronaphthalene-1-carboxylate (5) as a strating material, which was easily prepared on gram scale by baker's yeast-catalyzed asymmetric reduction.

Key words (-)-13-oxo-15,16-dinorlabda-8(17),11E-dien-19-oic acid; Thuja standishii; synthesis; (-)-1,4a-dimethyl-5-oxodecahydronaphthalene-1-carboxylate

A labdane-type diterpenoid, (-)-13-oxo-15,16-dinorlabda8(17),11E-dien-19-oic acid (1) has been reported by Tanaka to have potent cancer chemopreventive activity toward papilloma-bearing mice. ${ }^{1,2)}$ The acid (1) isolated from the stem bark of Thuja standishii (GoRD.) CARR. as a minor component had the most potent activity for the prevention of incipient carcinogenesis among the diterpenoids from the same plant source. Although two other plants, Platycladus orientaris $^{3)}$ and Juniperus chinensis, ${ }^{4,5)}$ were reported as a natural source of $\mathbf{1}$, its scarce supply from nature required efficient synthetic routes for further estimation as a cancer chemopreventive agent. Recently we published a chemical conversion from trans-communic acid (2), a major component of $T$. standishii, to 1 via allyl alcohol 3 (Fig. 1). $\left.{ }^{6}\right)$ Herein, as a part of our studies on chemoenzymatic synthesis of bioactive diterpenes, we would like to report an asymmetric total synthesis of methyl ester (4) of $\mathbf{1}$ via (-)-1,4a-dimethyl-5-oxodecahydronaphthalene $(\mathbf{5}),{ }^{7}$ which can be provided on gram scale from $\sigma$-symmetric 1,3-diketone $\mathbf{6}$ by using baker's yeast-catalyzed asymmetric reduction as a key step (Chart 1).

A synthetic approach was considered on the basis of the plausibility that decahydronaphthalene-1-carboxylate (5) was an excellent precursor for the synthesis of $\mathbf{4}$, and two routes (Routes A and B) were adopted as attainable strategies (Chart 1). The first strategy (Chart 1, Route A) was started with linking vinyl methyl ketone to the carbonyl carbon of $\mathbf{5}$, followed by introduction of the terminal methylene to an intermediate 7 . The second strategy (Chart 1, Route B) passed through aldehyde $\mathbf{8}(\mathrm{X}=\mathrm{O})$, of which the two carbonyl groups would be utilized to successively introduce a terminal

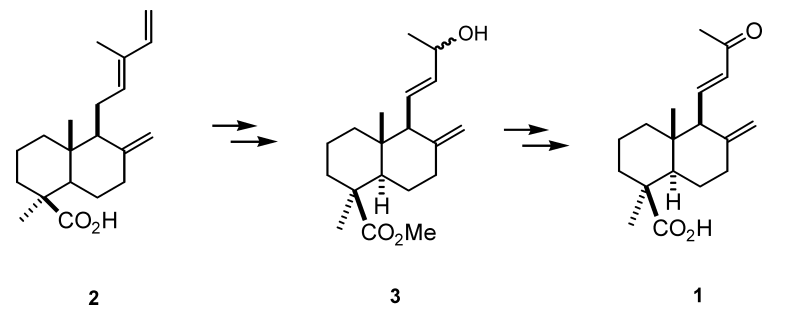

Fig. 1. Structures of Diterpenoids Related to $\mathbf{1}$ and trans-Communic Acid (2)

* To whom correspondence should be addressed.

e-mail: node@mb.kyoto-phu.ac.jp methylene and a propane unit by Wittig-type of reactions.

In order to achieve the former strategy (Route A), the precursor 5 was first derived to enol triflate (9) with $\mathrm{N}$-phenylbis(trifluoromethanesulfonimide) in the presence of potassium tert-butoxide in good yield (84\%) (Chart 2). The successive Heck reaction of $\mathbf{9}$ with methyl vinyl ketone catalyzed by palladium acetate $(2 \mathrm{~mol} \%)$ and triphenylphosphine resulted in a low yield (15\%) (Table 1, entry 1). When the reaction was run at higher temperature (Table 1, entries 2 , 3 ) or in the presence of a larger amount of the catalyst (Table 1 , entry 4), higher chemical yields (up to $78 \%$ ) were obtained. Moreover, the reaction employing bis(triphenylphosphine)palladium(II) chloride as a catalyst ${ }^{8}$ and running at $100{ }^{\circ} \mathrm{C}$ gave the best result ( $86 \%$ ) (Table 1 , entry 5 ).

Since we were able to obtain the coupled product $\mathbf{1 0}$ in good yield by using the Heck reaction, many efforts to introduce a terminal methylene were made. For example, direct conjugate addition of carbanion such as methoxymethyl cuprous magnesium bromide to the C-8 position of methyl ketone 10 was attempted in the beginning; however, all the trials resulted in failure. Thus, the conjugated methyl ketone

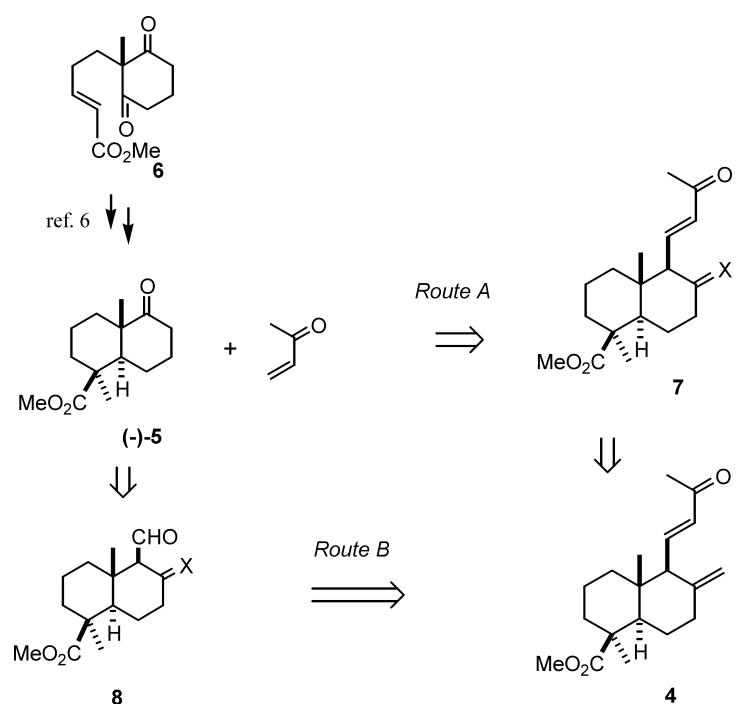

Chart 1. Synthetic Approach of $\mathbf{4}$ 


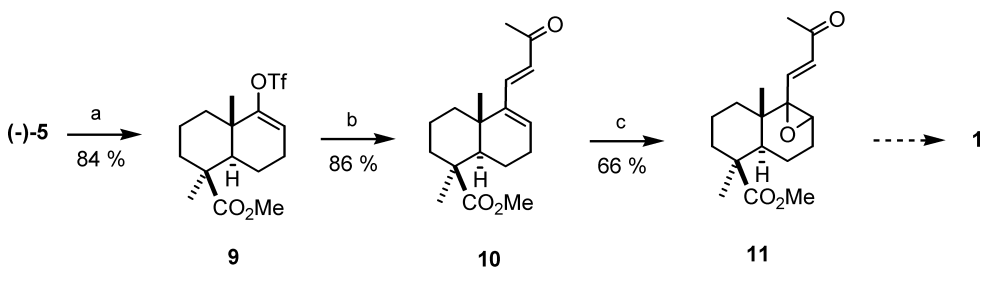

a: $t$-BuOK, PhNTf 2 , THF, b: methyl vinyl ketone, $\left(\mathrm{Ph}_{3} \mathrm{P}\right)_{2} \mathrm{PdCl}_{2}$, DMF, c: $m$-CPBA

Chart 2. Synthetic Route of $\mathbf{1}$ from (-)-5 using Heck Reaction as a Key Step

Table 1. Heck Reaction of Enol Triflate 9 with Methyl Vinyl Ketone

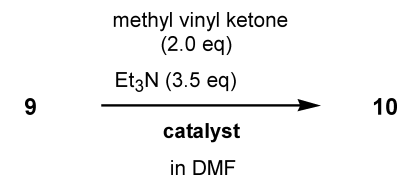

\begin{tabular}{|c|c|c|c|c|}
\hline Entry & Catalyst (mol\%) & Time $(\mathrm{h})$ & Temp. $\left({ }^{\circ} \mathrm{C}\right)$ & Yield (\%) \\
\hline 1 & $\mathrm{PPh}_{3}, \mathrm{Pd}(\mathrm{OAc})_{2}(2 \mathrm{~mol} \%)$ & 24 & r.t. & 15 \\
\hline 2 & $\mathrm{PPh}_{3}, \mathrm{Pd}(\mathrm{OAc})_{2}(2 \mathrm{~mol} \%)$ & 24 & 85 & 45 \\
\hline 3 & $\mathrm{PPh}_{3}, \mathrm{Pd}(\mathrm{OAc})_{2}(2 \mathrm{~mol} \%)$ & 24 & 100 & 51 \\
\hline 4 & $\mathrm{PPh}_{3}, \mathrm{Pd}(\mathrm{OAc})_{2}(20 \mathrm{~mol} \%)$ & 24 & 100 & 78 \\
\hline 5 & $\left(\mathrm{PPh}_{3}\right)_{2} \mathrm{PdCl}_{2}(10 \mathrm{~mol} \%)$ & 1.5 & 100 & 86 \\
\hline
\end{tabular}

10 was next derived to epoxide 11, which was attempted to transform diketone $7(\mathrm{X}=\mathrm{O})$ by Lewis acid-catalyzed hydride shift or was attacked by nucleophiles such as phenylthiomethyl magnesium bromide and methoxymethyl magnesium bromide. To our disappointment, none of them gave expected products (Chart 2).

After experiencing the difficulties in the Route A approach, the latter strategy (Chart 1, Route B) was attempted. In order to synthesize the key intermediate $\mathbf{8}$, introduction of a $\mathrm{C} 1$ unit at the carbonyl carbon of $\mathbf{5}$ by a Wittig-type reaction using methoxymethylene phosphonium ylide was tried. Surprisingly, the reaction did not afford the methyl enolate at all while Wittig reaction with methylidene triphenylphosphorane gave 12 in almost quantitative yield (99\%). Encouraged by the excellent result of the conventional Wittig reaction, the allylic methylene carbon of $\mathbf{1 2}$ was oxidized with selenium oxide in the presence of tert-butyl hydroperoxide ${ }^{9)}$ to afford allyl alcohol 13, of which the double bond was subjected to hydroboration-oxidation to yield diol 14. Stereochemistry of the secondary hydroxyl group of $\mathbf{1 4}$ was deduced on the basis of the spectral data of 2-hydroxydecahydro-5,5,8a-trimethyl-1-naphthalenemethanol (15) in the literature. ${ }^{10)}$ Although the oxidation of the two hydroxyl groups could formally afford the ketoaldehyde $8(X=O)$, the diol 14 was oxidized stepwise to carry out regioselective Wittig reaction for the two carbonyl groups of $\mathbf{8}(X=0)$. Therefore, the primary hydroxyl group of $\mathbf{1 4}$ was selectively protected with tert-butyldimethylsilyl chloride to afford $\mathbf{1 6}$. The remaining secondary hydroxyl group of $\mathbf{1 6}$ was then oxidized by DessMartin oxidation to give ketone 17, which was successively treated with the Wittig reagent to yield exo-olefin 18. Removal of the silyl protecting group of $\mathbf{1 8}$ with tetrabutylammonium fluoride led to primary alcohol $\mathbf{1 9}$, of which the successive oxidation with the Dess-Martin reagent afforded the key intermediate 20, which corresponds to $\mathbf{8}\left(\mathrm{X}=\mathrm{CH}_{2}\right)$
(Chart 3).

Finally, elongation of the 2-propanone side chain onto the aldehyde group of $\mathbf{2 0}$ was scrutinized (Table 2). Addition of an enolate prepared from acetone with lithium hexamethylsilazide did not give satisfactory results (Table 2, entries 1 , 2 ), while a better result to lead to the target molecule $4(45 \%)$ was obtained when aldehyde 19 was treated with triphenylphosphine ylide in refluxed toluene (Table 2, entry 4). All the spectral and physical data of our synthesized 4 completely coincided with that derived from naturally occurring 1.

It is noteworthy that the production of conjugate aldehyde 21 decreased the chemical yield of 4 . Unfortunately, neither direct saponification of the methyl ester in $\mathbf{4}$ nor demethylation with soft nucleophiles proceeded well due to the side reactions such as Michael addition to the conjugated ketone moiety and migration of the double bond at the terminal olefin. In order to avoid these undesired reactions, the synthetic route was revised so as to follow the same route from trans-communic acid (2) to $\mathbf{1}$. Namely, the carbonyl group of the conjugate ketone $\mathbf{4}$ was reduced to $\mathbf{3}$ under Luche's condition $^{11)}$ with cerium chloride and sodium borohydride and reoxidized after cleavage of the methyl ester with $n$-dodecanthiol and $n$-butyllithium in HMPA ${ }^{12,13)}$ via acid 22 (Chart 4).

In conclusion, we have succeeded in the formal asymmetric synthesis of optically pure (-)-13-oxo-15,16-dinorlabda$8(17), 11 E$-dien-19-oate (1). This synthetic route was started with (-)-1,4a-dimethyl-5-oxodecahydronaphthalene (5), which can be provided on gram scale by using baker's yeast-catalyzed asymmetric reduction. Therefore, our strategy is applicable for the synthesis of many derivatives of $\mathbf{1}$, which could support study on the structure-activity relationship for developing novel anti-tumor agents. Further studies are in progress.

\section{Experimental}

General Infrared (IR) spectra were recorded on a Shimadzu FTIR-8300 diffraction grating infrared spectrophotometer and ${ }^{1} \mathrm{H}-\mathrm{NMR}$ spectra were obtained on a JEOL JNM-AL300, or a Varian GEMINI 2000/200, or a Varian Unity INOVA-400 spectrometer with tetramethylsilane as an internal standard. ${ }^{13}$ C-NMR spectra were obtained on a Varian GEMINI 2000/200, or a Varian Unity INOVA-400 spectrometer with $\mathrm{CDCl}_{3}$ as an internal standard. Mass spectra (MS) were determined on a JEOL JMS-SX 102A QQ or a JEOL JMS-GC-mate mass spectrometer. Specific rotations were recorded on a Horiba SEPA-200 automatic digital polarimeter. Wakogel C-200 (silica gel) (100-200 mesh, Wako) was used for open column chromatography. Flash column chromatography was performed using Silica Gel 60N (Kanto Chemical Co., Inc.) as a solid support of the immobile phase. Kieselgel 60 F-254 plates (Merck) were used for thin-layer chromatography (TLC). Unless purification with silica gel gave a pure enough compound, the compounds were further treated with a recycle HPLC (JAI LC-908) on GPC column (JAIGEL $1 \mathrm{H}$ and $2 \mathrm{H}$ ). In the case it is possible, diastereomeric mix- 

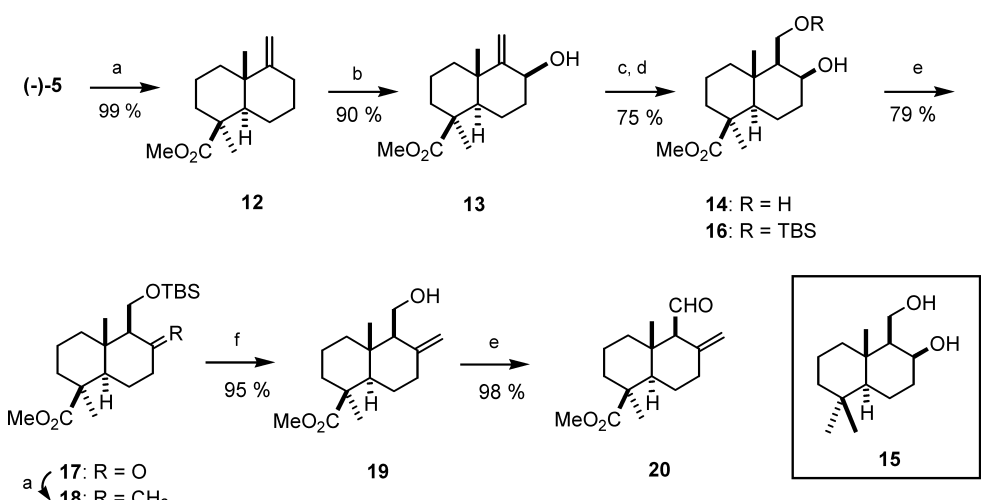

a: $\mathrm{Ph}_{3} \mathrm{P}=\mathrm{CH}_{2}$, b: $\mathrm{SeO}_{2}, t-\mathrm{BuO}_{2} \mathrm{H}, \mathrm{CH}_{2} \mathrm{Cl}_{2}, \mathbf{c}: \mathrm{BH}_{3} . \mathrm{SMe}_{2}$, THF, then $\mathrm{H}_{2} \mathrm{O}_{2}, \mathrm{NaOH}$,

d: $\mathrm{TBSCl}, \mathrm{Et}_{3} \mathrm{~N}, \mathrm{CH}_{2} \mathrm{Cl}_{2}$, e: Dess-Martin reagent, f: TBAF, THF

Chart 3. Synthesis of the Key Intermediate (20) from (-)-5

Table 2. Introduction of a Propane Unit to the Aldehyde 20

\begin{tabular}{|c|c|c|c|c|c|c|}
\hline & & & 4 & & & \\
\hline Entry & C3 unit & Base & Solvent & Temp. & Time (h) & Yield (\%) \\
\hline 1 & Acetone & LHMDS & THF & $-78^{\circ} \mathrm{C}$-r.t. & 18 & 14 \\
\hline 2 & Acetone & LHMDS & THF & $-78^{\circ} \mathrm{C}$-r.t. & 24 & 29 \\
\hline 3 & $(\mathrm{EtO})_{2} \mathrm{P}(\mathrm{O}) \mathrm{CH}_{2} \mathrm{COCH}_{3}$ & $n$-BuLi & THF & $0^{\circ} \mathrm{C}-$ r.t. & 3.5 & 31 \\
\hline 4 & $\mathrm{Ph}_{3} \mathrm{P}=\mathrm{CHCOCH}_{3}$ & & Toluene & Reflux & 18 & 45 \\
\hline 5 & $\mathrm{Ph}_{3} \mathrm{P}=\mathrm{CHCOCH}_{3}$ & & DMF & $130^{\circ} \mathrm{C}$ & 48 & - \\
\hline
\end{tabular}

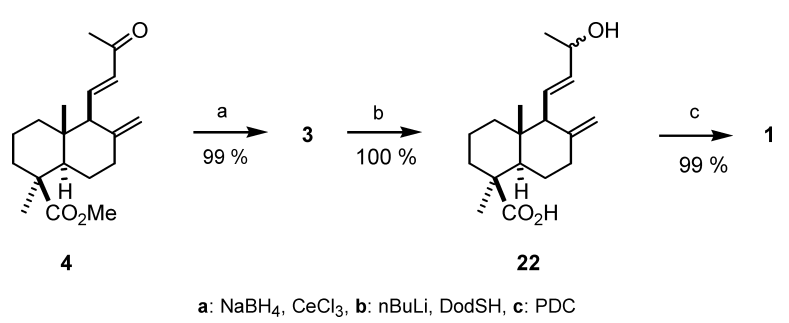

Chart 4. Synthesis of 1 from 4

tures were also separated by a recycle HPLC (JAI LC-908) on silica gel column (Kusano Si-10) after the purification mentioned above.

Methyl 1,4a-Dimethyl-5-trifluoromethanesulfonyl-1,2,3,4,4a,7,8,8a-octahydronaphthalene-1-carboxylate (9) Potassium tert-butoxide $(74 \mathrm{mg}$, $0.66 \mathrm{mmol}$ ) was added to a solution of $(-)-5(79 \mathrm{mg}, 0.33 \mathrm{mmol})$ in tetrahydrofuran $(3 \mathrm{ml})$ at $-78^{\circ} \mathrm{C}$ and the mixture was stirred for $20 \mathrm{~min}$. $N$-Phenylbis(trifluoromethanesulfonimide) $(249 \mathrm{mg}, 0.66 \mathrm{mmol})$ was added to the reaction mixture, which was stirred for another $20 \mathrm{~min}$ with keeping the temperature. After the reaction, the reaction mixture was poured into a saturated aqueous solution of ammonium chloride and extracted with diethyl ether The organic layer was washed with a saturated aqueous solution of sodium chloride, dried over magnesium sulfate, and condensed in vacuo. The residue was purified by silica gel column chromatography (hexane : ethyl acetate $=5: 1)$ to afford $9(103 \mathrm{mg}, 84 \%)$ as colorless oil. ${ }^{1} \mathrm{H}-\mathrm{NMR}(300 \mathrm{MHz}$, $\left.\mathrm{CDCl}_{3}\right) \delta: 0.92-1.13(1 \mathrm{H}, \mathrm{m}), 0.96(3 \mathrm{H}, \mathrm{s}), 1.18-1.38(2 \mathrm{H}, \mathrm{m}), 1.23(3 \mathrm{H}$ s), $1.46(2 \mathrm{H}, \mathrm{m}), 1.75-1.87(2 \mathrm{H}, \mathrm{m}), 2.00-2.28(4 \mathrm{H}, \mathrm{m}), 3.66(3 \mathrm{H}, \mathrm{s})$ $5.62-5.64(1 \mathrm{H}, \mathrm{m}) .{ }^{13} \mathrm{C}-\mathrm{NMR}\left(100 \mathrm{MHz}, \mathrm{CDCl}_{3}\right) \delta: 17.0,18.7,19.7,24.9$, $28.2,34.6,37.4,39.6,43.4,51.4,52.6,115.5,128.0,156.1,177.1$. IR $\left(\mathrm{CHCl}_{3}\right) \mathrm{cm}^{-1}: 2951,2361,2341,1720,1408,1238,1207,1142$. EI-MS $\mathrm{m} / \mathrm{z}$ 370.1051 (Calcd for $\left.\mathrm{C}_{15} \mathrm{H}_{21} \mathrm{~F}_{3} \mathrm{O}_{5} \mathrm{~S}: 370.1061\right)$. MS $(70 \mathrm{eV}) \mathrm{m} / \mathrm{z}: 370\left(\mathrm{M}^{+}, 2\right)$, 295 (15), 237 (28), 205 (18), 177 (30), 161 (100), 145 (38), 105 (46), 91
(31), 67 (23), 55 (42).

Methyl 1,4a-Dimethyl-5-(3-oxobutenyl)-1,2,3,4,4a,7,8,8a-octahydronaphthalene-1-carboxylate (10) Methyl vinyl ketone (199 $\mu$, $2.45 \mathrm{mmol}$ ), bis(triphenylphosphine)palladium(II) chloride $(17 \mathrm{mg}$, $0.024 \mathrm{mmol})$, and triethylamine $(569 \mu \mathrm{l}, 4.07 \mathrm{mmol})$ were added to a solution of $9(431 \mathrm{mg}, 1.16 \mathrm{mmol})$ in $N, N$-dimethylformamide $(5 \mathrm{ml})$ and the mixture was stirred for $3 \mathrm{~h}$ at $75^{\circ} \mathrm{C}$. After the reaction, the organic solvent was removed in vacuo and the residue was purified by silica gel column chromatography (hexane : ethyl acetate $=6: 1)$ to afford $\mathbf{1 0}(277 \mathrm{mg}, 82 \%)$ as colorless amorphous. ${ }^{1} \mathrm{H}-\mathrm{NMR}\left(300 \mathrm{MHz}, \mathrm{CDCl}_{3}\right) \delta: 0.92(3 \mathrm{H}, \mathrm{s}), 1.00$ $1.40(4 \mathrm{H}, \mathrm{m}), 1.23(3 \mathrm{H}, \mathrm{s}), 1.52-1.58(2 \mathrm{H}, \mathrm{m}), 1.74-2.34(5 \mathrm{H}, \mathrm{m}), 2.25$ $(3 \mathrm{H}, \mathrm{s}), 3.66(3 \mathrm{H}, \mathrm{s}), 6.04-6.06(1 \mathrm{H}, \mathrm{m}), 6.28(1 \mathrm{H}, \mathrm{d}, J=15.8 \mathrm{~Hz}), 7.17$ $(1 \mathrm{H}, \mathrm{d}, J=15.8 \mathrm{~Hz}) .{ }^{13} \mathrm{C}-\mathrm{NMR}\left(100 \mathrm{MHz}, \mathrm{CDCl}_{3}\right) \delta: 18.4,19.3,20.0,27.4$, $28.1,28.4,37.3,37.6,38.2,43.8,51.3,52.8,127.5,128.9,143.6,144.5$, 177.7, 198.5. IR $\left(\mathrm{CHCl}_{3}\right) \mathrm{cm}^{-1}: 3009,2951,1720,1666,1593,1465,1362$, 1258, 1238, 1177, 1153. EI-MS $\mathrm{m} / \mathrm{z}$ : 290.1880 (Calcd for $\mathrm{C}_{18} \mathrm{H}_{26} \mathrm{O}_{3}$ : 290.1882). MS (70 eV) m/z: $290\left(\mathrm{M}^{+}, 2\right), 275$ (9), 231 (11), 215 (23), 187 (21), 159 (22), 121 (100), 108 (82), 91 (29), 55 (21).

Methyl 4,7a-Dimethyl-7b-(3-oxobutenyl)-decahydro-1-oxa-cyclopropa[a]naphthalene-4-carboxylate (11) A saturated aqueous solution of sodium bicarbonate $(1.5 \mathrm{ml})$ was added to a solution of $\mathbf{1 0}(28 \mathrm{mg}$, $0.096 \mathrm{mmol})$ and $m$-chloroperbenzoic acid $(80 \%)(25 \mathrm{mg}, 0.12 \mathrm{mmol})$ in dichloromethane $(2 \mathrm{ml})$ and the biphasic mixture was stirred vigorously for $2 \mathrm{~h}$ at $0{ }^{\circ} \mathrm{C}$. After the reaction, the reaction mixture was extracted with ethyl acetate. The organic layer was washed with a saturated aqueous solution of sodium chloride, dried over, and condensed in vacuo. The residue was purified by silica gel column chromatography (hexane : ethyl acetate $=8: 1$ ) to afford two diastereomers of 11 (11a, b) $(19 \mathrm{mg}, 66 \%)$ as colorless crystals, which were separated by HPLC on silica gel column.

11a: ${ }^{1} \mathrm{H}-\mathrm{NMR}\left(400 \mathrm{MHz}, \mathrm{CDCl}_{3}\right) \delta: 0.86(3 \mathrm{H}, \mathrm{s}), 1.00-1.14(2 \mathrm{H}, \mathrm{m})$, $1.17(3 \mathrm{H}, \mathrm{s}), 1.47(6 \mathrm{H}, \mathrm{m}), 1.84-1.97(1 \mathrm{H}, \mathrm{m}), 2.17-2.28(2 \mathrm{H}, \mathrm{m}), 2.24$ $(3 \mathrm{H}, \mathrm{s}), 2.97(1 \mathrm{H}, \mathrm{s}), 3.63(3 \mathrm{H}, \mathrm{s}), 6.28(1 \mathrm{H}, \mathrm{d}, J=15.4 \mathrm{~Hz}), 7.12(1 \mathrm{H}, \mathrm{d}$, $J=15.4 \mathrm{~Hz}) .{ }^{13} \mathrm{C}-\mathrm{NMR}\left(100 \mathrm{MHz}, \mathrm{CDCl}_{3}\right) \delta: 14.8,17.7,19.9,28.3,28.4$, 28.7, 37.9, 38.2, 39.0, 44.0, 51.6, 53.6, 64.8, 66.4, 129.3, 144.4, 177.7, 
198.3. IR $\left(\mathrm{CHCl}_{3}\right) \mathrm{cm}^{-1}: 3036,3009,2980,2949,2853,1719,1676,1624$, $1468,1437,1360,1290,1238,1155$. EI-MS m/z: 306.1826 (Calcd for $\left.\mathrm{C}_{18} \mathrm{H}_{26} \mathrm{O}_{4}: 306.1831\right)$. MS (70 eV) m/z: $306\left(\mathrm{M}^{+}, 2\right), 288$ (2), 263 (3), 213 (2), 181 (4), 175 (8), 148 (100), 135 (60), $121(60), 109$ (27), 105 (30), 91 (35), 67 (27), 55 (51).

11b: ${ }^{1} \mathrm{H}-\mathrm{NMR}\left(400 \mathrm{MHz}, \mathrm{CDCl}_{3}\right) \delta: 1.00-1.10(1 \mathrm{H}, \mathrm{m}), 1.04(3 \mathrm{H}, \mathrm{s})$, $1.17(3 \mathrm{H}, \mathrm{s}), 1.37-1.56(3 \mathrm{H}, \mathrm{m}), 1.60-1.96(5 \mathrm{H}, \mathrm{m}), 2.02-2.11(1 \mathrm{H}, \mathrm{m})$, $2.13-2.21(1 \mathrm{H}, \mathrm{m}), 2.24(3 \mathrm{H}, \mathrm{s}), 2.93(1 \mathrm{H}, \mathrm{d}, J=4.8 \mathrm{~Hz}), 3.66(3 \mathrm{H}, \mathrm{s}), 6.29$ $(1 \mathrm{H}, \mathrm{d}, J=15.4 \mathrm{~Hz}), 7.12(1 \mathrm{H}, \mathrm{d}, J=15.4 \mathrm{~Hz}) .{ }^{13} \mathrm{C}-\mathrm{NMR}\left(100 \mathrm{MHz}, \mathrm{CDCl}_{3}\right)$ $\delta: 16.2,18.2,24.1,28.3,28.8,35.9,36.9,37.5,43.4,45.6,51.3,63.8,67.2$, 130.0, 143.2, 177.8, 197.9. IR $\left(\mathrm{CHCl}_{3}\right) \mathrm{cm}^{-1}: 3036,3007,2980,2951,2880$, $1719,1674,1626,1565,1435,1360,1263,1240,1150$. EI-MS $\mathrm{m} / \mathrm{z}$ : 306.1828 (Calcd for $\mathrm{C}_{18} \mathrm{H}_{26} \mathrm{O}_{4}: 306.1831$ ). MS $(70 \mathrm{eV}) \mathrm{m} / \mathrm{z}: 306\left(\mathrm{M}^{+}, 2\right)$, 288 (2), 263 (3), 213 (3), 175 (8), 148 (100), 135 (57), 121(47), 109 (25), 105 (31), 91 (34), 55 (39).

(-)-Methyl 1,4a-Dimethyl-5-methylenedecahydronaphthalene-1-carboxylate (12) Potassium tert-butoxide $(814 \mathrm{mg}, 7.25 \mathrm{mmol})$ was added to a suspension of methyltriphenylphosphonium bromide $(2.59 \mathrm{~g}, 7.25 \mathrm{mmol})$ in terahydrofuran $(25 \mathrm{ml})$ and the mixture was stirred for $5 \mathrm{~min}$ at room temperature. And then, (-) -5 (576 mg, $2.42 \mathrm{mmol})$ was added to the mixture, which was stirred for $30 \mathrm{~min}$ at $60^{\circ} \mathrm{C}$. After the reaction, the reaction mixture was poured into a saturated aqueous solution of ammonium chloride and extracted with diethyl ether. The organic layer was successively washed with a saturated aqueous solution of sodium chloride and distilled water, dried over magnesium sulfate, and condensed in vacuo. The residue was purified by silica gel column chromatography (hexane : ethyl acetate $=8: 1$ ) to afford $12(564 \mathrm{mg}, 99 \%)$ as a colorless oil. ${ }^{1} \mathrm{H}-\mathrm{NMR}\left(400 \mathrm{MHz}, \mathrm{CDCl}_{3}\right) \delta$ : $0.88(3 \mathrm{H}, \mathrm{s}), 1.04(1 \mathrm{H}, \mathrm{dt}, J=13.5,4.0 \mathrm{~Hz}), 1.15-1.27(2 \mathrm{H}, \mathrm{m}), 1.16(3 \mathrm{H}$ s), $1.46-1.60(2 \mathrm{H}, \mathrm{m}), 1.66-1.71(1 \mathrm{H}, \mathrm{m}), 1.85-1.96(4 \mathrm{H}, \mathrm{m}), 2.09-$ $2.21(2 \mathrm{H}, \mathrm{m}), 2.26-2.34(1 \mathrm{H}, \mathrm{m}), 3.66(3 \mathrm{H}, \mathrm{s}), 4.57(1 \mathrm{H}, \mathrm{m}), 4.58(1 \mathrm{H}, \mathrm{m})$ ${ }^{13} \mathrm{C}-\mathrm{NMR}\left(100 \mathrm{MHz}, \mathrm{CDCl}_{3}\right) \delta: 18.5,19.6,23.9,28.8,29.1,33.5,37.5$, $38.3,40.2,44.4,51.2,55.2,104.3,159.2,177.9$. IR $\left(\mathrm{CHCl}_{3}\right) \mathrm{cm}^{-1}: 2939$, 2854, 1717, 1632, 1447, 1337. EI-MS $m / z: 238.1776$ (Calcd for $\mathrm{C}_{15} \mathrm{H}_{24} \mathrm{O}_{2}$ : 236.1775). MS (70 eV) m/z: $236\left(\mathrm{M}^{+}, 99\right), 204$ (37), 176 (95), 161 (55), 121 (100), 107 (97), 95 (96), 81 (53), 67 (53), 55 (52). $[\alpha]_{\mathrm{D}}^{25}=-51.5^{\circ}(c=0.56$ $\mathrm{CHCl}_{3}$ ). Anal. Calcd for $\mathrm{C}_{15} \mathrm{H}_{24} \mathrm{O}_{2}: \mathrm{C}, 76.23 ; \mathrm{H}, 10.24$. Found: $\mathrm{C}, 75.96 ; \mathrm{H}$, 10.27 .

(-)-Methyl 6-Hydroxy-1,4a-dimethyl-5-methylenedecahydronaphthalene-1-carboxylate (13) Selenium oxide $(139 \mathrm{mg}, 1.26 \mathrm{mmol})$ and tertbutyl hydroperoxide ( $70 \mathrm{wt} \%$ in water, $700 \mu 1,5.03 \mathrm{mmol}$ ) were added to a solution of $12(634 \mathrm{mg}, 2.51 \mathrm{mmol})$ in dichloromethane $(15 \mathrm{ml})$ and the mixture was stirred for $24 \mathrm{~h}$ at room temperature. After the reaction, the reaction mixture was poured into a saturated aqueous solution of sodium thiosulfate and extracted with chloroform. The organic layer was dried over magnesium sulfate and condensed in vacuo. The residue was purified by silica gel column chromatography (hexane : ethyl acetate $=10: 1 \rightarrow 2: 1$ ) to afford 12 ( $259 \mathrm{mg}, 44 \%$ rec.) and $\mathbf{1 3}$ [326 mg, 51\% (conversion yield: 91\%)] as colorless needles. mp. $103-104{ }^{\circ} \mathrm{C}$ (hexane/ethyl acetate). ${ }^{1} \mathrm{H}-\mathrm{NMR}(400 \mathrm{MHz}$ $\left.\mathrm{CDCl}_{3}\right) \delta: 1.02(1 \mathrm{H}, \mathrm{dt}, J=13.5,4.1 \mathrm{~Hz}), 1.08(3 \mathrm{H}, \mathrm{s}), 1.16-1.21(1 \mathrm{H}, \mathrm{m})$, $1.19(3 \mathrm{H}, \mathrm{s}), 1.40-1.58(4 \mathrm{H}, \mathrm{m}), 1.76-1.82(2 \mathrm{H}, \mathrm{m}), 1.88-2.06(2 \mathrm{H}, \mathrm{m})$ $2.18-2.28(2 \mathrm{H}, \mathrm{m}), 3.67(3 \mathrm{H}, \mathrm{s}), 4.35(1 \mathrm{H}, \mathrm{t}, J=2.9 \mathrm{~Hz}), 4.86(1 \mathrm{H}, \mathrm{d}$, $J=1.1 \mathrm{~Hz}), 4.91(1 \mathrm{H}, \mathrm{d}, J=1.1 \mathrm{~Hz}) .{ }^{13} \mathrm{C}-\mathrm{NMR}\left(100 \mathrm{MHz}, \mathrm{CDCl}_{3}\right) \delta: 18.3$ $19.1,20.5,28.7,34.8,38.1,38.2,39.8,44.2,51.3,55.0,74.8,111.1,159.3$ 177.9. IR $\left(\mathrm{CHCl}_{3}\right) \mathrm{cm}^{-1}: 3009,2991,2939,2856,1717,1466,1437,1236$ EI-MS $m / z: 252.1725\left(\right.$ Calcd for $\left.\mathrm{C}_{15} \mathrm{H}_{24} \mathrm{O}_{3}: 252.1726\right)$. MS $(70 \mathrm{eV}) \mathrm{m} / \mathrm{z}: 252$ $\left(\mathrm{M}^{+}, 6\right), 237$ (12), 192 (13), 175 (95), 159 (27), 145 (19), 123 (100), 109 (48), $105(63), 91(54), 67(46), 55(74) .[\alpha]_{\mathrm{D}}^{25}=-1.30^{\circ}\left(c=0.61, \mathrm{CHCl}_{3}\right)$. Anal. Calcd for $\mathrm{C}_{15} \mathrm{H}_{24} \mathrm{O}_{3}: \mathrm{C}, 71.39 ; \mathrm{H}, 9.59$. Found: $\mathrm{C}, 71.27 ; \mathrm{H}, 9.79$.

(+)-Methyl 6-Hydroxy-5-hydroxymethyl-1,4a-dimethyldecahydronaphthalene-1-carboxylate (14) A borane dimethyl sulfide complex ( $2.0 \mathrm{M}$ solution in tetrahydrofuran, $436 \mu 1,0.87 \mathrm{mmol}$ ) was added to a solution of $13(220 \mathrm{mg}, 0.87 \mathrm{mmol})$ in tetrahydrofuran $(5 \mathrm{ml})$ and the mixture was stirred for $2 \mathrm{~h}$ at room temperature. And then, hydrogen peroxide (30 $\mathrm{wt} \%$ solution in water, $1 \mathrm{ml}$ ) and an aqueous solution of sodium hydroxide $(1.0 \mathrm{M}, 1 \mathrm{ml})$ were added to the reaction mixture, which was stirred for $15 \mathrm{~min}$. After the reaction, the mixture was poured into saturated aqueous solution of ammonium chloride and extracted with ethyl acetate. The organic layer was washed with a saturated aqueous solution of sodium chloride, dried over magnesium sulfate, and condensed in vacuo. The residue was purified by silica gel column chromatography (hexane: ethyl acetate $=1: 1)$ to afford $\mathbf{1 3}(16 \mathrm{mg}, 7 \%$ rec.) and $\mathbf{1 4}[199 \mathrm{mg}, 85 \%$ (conversion yield: $91 \%$ )] as colorless crystals. mp. $116-118^{\circ} \mathrm{C}$ (hexane/ethyl acetate). ${ }^{1} \mathrm{H}-\mathrm{NMR}\left(400 \mathrm{MHz}, \mathrm{CDCl}_{3}\right) \delta: 0.93(3 \mathrm{H}, \mathrm{s}), 1.03(1 \mathrm{H}, \mathrm{dt}, J=13.4$, $4.0 \mathrm{~Hz}), 1.11-1.20(2 \mathrm{H}, \mathrm{m}), 1.20(3 \mathrm{H}, \mathrm{s}), 1.41-1.57(2 \mathrm{H}, \mathrm{m}), 1.75-2.13$
$(7 \mathrm{H}, \mathrm{m}), 2.16-2.20(1 \mathrm{H}, \mathrm{m}), 3.65(3 \mathrm{H}, \mathrm{s}), 3.83-3.87(1 \mathrm{H}, \mathrm{m}), 3.95-4.00$ $(1 \mathrm{H}, \mathrm{m}), 4.27\left(1 \mathrm{H}\right.$, br s) ${ }^{13} \mathrm{C}-\mathrm{NMR}\left(100 \mathrm{MHz}, \mathrm{CDCl}_{3}\right) \delta: 18.3,19.1,20.5$, 28.7, 34.8, 38.1, 38.2, 39.8, 44.2, 51.3, 55.0, 74.8, 111.1, 159.3, 177.9. IR $\left(\mathrm{CHCl}_{3}\right) \mathrm{cm}^{-1}: 3618,3487,3435,3028,2999,2951,2937,1717,1468$, 1450, 1435. EI-MS m/z: 270.1831 (Calcd for $\mathrm{C}_{15} \mathrm{H}_{26} \mathrm{O}_{4}: 270.1829$ ). MS (70 eV) m/z: $270\left(\mathrm{M}^{+}, 1\right), 252$ (5), 238 (18), 222 (14), 193 (20), 175 (37), 161 (24), 147 (20), 121 (100), 109 (58), 93 (55), 81 (76), 67 (57), 55 (83). $[\alpha]_{\mathrm{D}}^{25}=+35.6^{\circ}\left(c=2.15, \mathrm{CHCl}_{3}\right)$. Anal. Calcd for $\mathrm{C}_{15} \mathrm{H}_{26} \mathrm{O}_{4}: \mathrm{C}, 66.64 ; \mathrm{H}$, 9.69. Found: C, 66.61; H, 9.60.

(+)-Methyl 5-(tert-Butyldimethylsilyloxymethyl)-6-hydroxy-1,4a-dimethyldecahydronaphthalene-1-carboxylate (16) tert-Butyldimethylsilyl chloride $(100 \mathrm{mg}, 0.64 \mathrm{mmol})$ and triethylamine $(167 \mathrm{ml}, 1.20 \mathrm{mmol})$ were added to a solution of $\mathbf{1 4}(108 \mathrm{mg}, 0.40 \mathrm{mmol})$ in dichloromethane $(5 \mathrm{ml})$ and the mixture was stirred for $18 \mathrm{~h}$ at room temperature. After the reaction, the mixture was poured into a saturated aqueous solution of ammonium chloride and extracted with ethyl acetate. The organic layer was washed with a saturated aqueous solution of sodium chloride, dried over magnesium sulfate, and condensed in vacuo. The residue was purified by silica gel column chromatography (hexane : ethyl acetate $=5: 1)$ to afford $\mathbf{1 6}(135 \mathrm{mg}, 88 \%)$ as a colorless oil. ${ }^{1} \mathrm{H}-\mathrm{NMR}\left(400 \mathrm{MHz}, \mathrm{CDCl}_{3}\right) \delta: 0.076(3 \mathrm{H}, \mathrm{s}), 0.079(3 \mathrm{H}, \mathrm{s})$, $0.89(9 \mathrm{H}, \mathrm{s}), 0.94-1.12(3 \mathrm{H}, \mathrm{m}), 0.99(3 \mathrm{H}, \mathrm{s}), 1.19(3 \mathrm{H}, \mathrm{s}), 1.23-1.35$ $(2 \mathrm{H}, \mathrm{m}), 1.40-1.48(2 \mathrm{H}, \mathrm{m}), 1.73-2.00(4 \mathrm{H}, \mathrm{m}), 2.06-2.20(2 \mathrm{H}, \mathrm{m}), 3.64$ $(3 \mathrm{H}, \mathrm{s}), 3.80(1 \mathrm{H}, \mathrm{dd}, J=10.4,4.2 \mathrm{~Hz}), 4.03(1 \mathrm{H}, \mathrm{dd}, J=10.4,4.9 \mathrm{~Hz})$, $4.22-4.23(1 \mathrm{H}, \mathrm{m}) .{ }^{13} \mathrm{C}-\mathrm{NMR}\left(100 \mathrm{MHz}, \mathrm{CDCl}_{3}\right) \delta:-5.7,-5.6,15.6$, $18.0,18.6,18.8,25.8$ (3), 28.7, 34.8, 37.8, 38.2, 40.0, 43.6, 51.2, 54.1, 56.7, 62.8, 69.8, 178.0. IR $\left(\mathrm{CHCl}_{3}\right) \mathrm{cm}^{-1}: 3672,3468,3024,3001,2953,2932$, $1715,1472,1450,1436$. CI-MS $m / z: 385.2774$ (Calcd for $\mathrm{C}_{21} \mathrm{H}_{41} \mathrm{O}_{4} \mathrm{Si}$ : 385.2775). MS $m / z: 385\left(\mathrm{M}^{+}+\mathrm{H}, 3\right), 367$ (8), 327 (3), 251 (4), 235 (100), $175(85), 119(8), 105(11), 75(17), 57(72) .[\alpha]_{\mathrm{D}}^{25}=+43.1^{\circ}(c=0.74$, $\left.\mathrm{CHCl}_{3}\right)$.

(-)-Methyl 5-(tert-Butyldimethylsilyloxymethyl)-6-oxo-1,4a-dimethyldecahydronaphthalene-1-carboxylate (17) Dess-Martin periodinane $(1.69 \mathrm{~g}, 3.97 \mathrm{mmol})$ was added to a solution of $16(953 \mathrm{mg}, 2.48 \mathrm{mmol})$ in dichloromethane $(35 \mathrm{ml})$ at $0{ }^{\circ} \mathrm{C}$ and the mixture was stirred for $5 \mathrm{~h}$ at room temperature. After the reaction, the reaction mixture was poured into a saturated aqueous solution of sodium thiosulfate and extracted with diethyl ether. The organic layer was successively washed with a saturated aqueous solution of sodium bicarbonate and sodium chloride, dried over magnesium sulfate and condensed in vacuo. The residue was purified by silica gel column chromatography (hexane : ethyl acetate $=5: 1)$ to afford $17(915 \mathrm{mg}$, $97 \%)$ as a colorless oil. ${ }^{1} \mathrm{H}-\mathrm{NMR}\left(200 \mathrm{MHz}, \mathrm{CDCl}_{3}\right) \delta: 0.034(3 \mathrm{H}, \mathrm{s}), 0.054$ $(3 \mathrm{H}, \mathrm{s}), 0.55(3 \mathrm{H}, \mathrm{s}), 0.86(9 \mathrm{H}, \mathrm{s}), 1.12(1 \mathrm{H}, \mathrm{dt}, J=13.2,3.9 \mathrm{~Hz}), 1.27(3 \mathrm{H}$, s), $1.34-1.56(2 \mathrm{H}, \mathrm{m}), 1.67-1.90(3 \mathrm{H}, \mathrm{m}), 2.09-2.42(6 \mathrm{H}, \mathrm{m}), 3.58(1 \mathrm{H}$ dd, $J=10.4,4.0 \mathrm{~Hz}), 3.63(3 \mathrm{H}, \mathrm{s}), 4.04(1 \mathrm{H}, \mathrm{dd}, J=10.4,6.6 \mathrm{~Hz}) .{ }^{13} \mathrm{C}-\mathrm{NMR}$ $\left(50 \mathrm{MHz}, \mathrm{CDCl}_{3}\right) \delta:-5.4,-5.2,13.8,18.3,19.7,25.8,26.0(3), 28.9,38.1$, 39.7, 43.0, 44.3, 51.4, 54.9, 57.1, 65.5, 177.0, 210.3. IR $\left(\mathrm{CHCl}_{3}\right) \mathrm{cm}^{-1}$ : $3690,3030,2993,2952,2931,1715,1653,1603,1472,1435$. EI-MS $\mathrm{m} / z$ : 382.2539 (Calcd for $\left.\mathrm{C}_{21} \mathrm{H}_{38} \mathrm{O}_{4} \mathrm{Si}: 382.2540\right)$. MS $(70 \mathrm{eV}) \mathrm{m} / \mathrm{z}: 382\left(\mathrm{M}^{+}, 1\right)$, 325 (32), 293 (7), 265 (9), 233 (7), 213 (7), 181 (9), 173 (53), 157 (100), $121(92), 105(20), 91(26), 75(55), 55(21) .[\alpha]_{D}^{25}=-15.8^{\circ}(c=0.53$, $\mathrm{CHCl}_{3}$ ).

(+)-Methyl 5-(tert-Butyldimethylsilyloxymethyl)-6-methylene-1,4a-dimethyldecahydronaphthalene-1-carboxylate (18) $n$-Butyllitium $(2.60 \mathrm{M}$ solution in $n$-hexane, $1.9 \mathrm{ml}, 4.93 \mathrm{mmol}$ ) was added to a suspension of methyltriphenylphosphonium bromide $(2.11 \mathrm{~g}, 5.92 \mathrm{mmol})$ in tetrahydrofuran $(30 \mathrm{ml})$ at $0{ }^{\circ} \mathrm{C}$ and the mixture was stirred for $30 \mathrm{~min}$ at room temperature. And then, a solution of $17(755 \mathrm{mg}, 1.97 \mathrm{mmol})$ in tetrahydrofuran $(10 \mathrm{ml})$ was added to the reaction mixture, which was stirred for another $5 \mathrm{~h}$ After the reaction, the mixture was poured into a saturated aqueous solution of ammonium chloride and extracted with ethyl acetate. The organic layer was washed with a saturated aqueous solution of sodium chloride, dried over magnesium sulfate, and condensed in vacuo. The residue was purified by silica gel column chromatography (hexane : diethyl ether $=50: 1$ ) to afford $\mathbf{1 8}$ $(612 \mathrm{mg}, 82 \%)$ as a colorless oil. ${ }^{1} \mathrm{H}-\mathrm{NMR}\left(400 \mathrm{MHz}, \mathrm{CDCl}_{3}\right) \delta: 0.034(6 \mathrm{H}$ s), $0.57(3 \mathrm{H}, \mathrm{s}), 0.87(9 \mathrm{H}, \mathrm{s}), 1.06(1 \mathrm{H}, \mathrm{dt}, J=13.3,4.2 \mathrm{~Hz}), 1.18(3 \mathrm{H}, \mathrm{s})$, $1.21-1.34(2 \mathrm{H}, \mathrm{m}), 1.48-1.54(1 \mathrm{H}, \mathrm{m}), 1.71-1.86(4 \mathrm{H}, \mathrm{m}), 1.93-2.00$ $(2 \mathrm{H}, \mathrm{m}), 2.14-2.20(1 \mathrm{H}, \mathrm{m}), 2.36-2.41(1 \mathrm{H}, \mathrm{m}), 3.62(3 \mathrm{H}, \mathrm{s}), 3.75(1 \mathrm{H}$, dd, $J=10.4,7.4 \mathrm{~Hz}), 3.84(1 \mathrm{H}, \mathrm{dd}, J=10.4,3.8 \mathrm{~Hz}), 4.64(1 \mathrm{H}, \mathrm{m}), 4.85(1 \mathrm{H}$ m). ${ }^{13} \mathrm{C}-\mathrm{NMR}\left(100 \mathrm{MHz}, \mathrm{CDCl}_{3}\right) \delta:-5.4,-5.3,13.5,18.2,19.9,25.8,25.9$ (3), 28.9, 38.2, 38.4, 39.4, 39.5, 44.1, 51.2, 56.2, 57.6, 59.8, 107.2, 147.2, 177.8. IR $\left(\mathrm{CHCl}_{3}\right) \mathrm{cm}^{-1}: 3690,3036,2991,2932,1717,1645,1603,1472$, 1450. EI-MS $m / z$ : 380.2747 (Calcd for $\left.\mathrm{C}_{22} \mathrm{H}_{40} \mathrm{O}_{3} \mathrm{Si}: 380.2738\right)$. MS $(20 \mathrm{eV})$ m/z: $380\left(\mathrm{M}^{+}, 1\right), 365$ (1), 323 (100), 291 (7), 281 (4), 263 (7), 207 (11), $189(48), 159(12), 147(10), 133(8), 105(7), 75(16) .[\alpha]_{D}^{25}=+30.3^{\circ}$ 
$\left(c=0.85, \mathrm{CHCl}_{3}\right)$

(+)-Methyl 5-Hydroxymethyl-6-methylene-1,4a-dimethyldecahydronaphthalene-1-carboxylate (19) Tetra- $n$-butylammonium fluoride $(1.0 \mathrm{M}$ solution in tetrahydrofuran, $2.3 \mathrm{ml}, 2.26 \mathrm{mmol}$ ) was added to a solution of $\mathbf{1 8}$ $(574 \mathrm{mg}, 1.51 \mathrm{mmol})$ in tetrahydrofuran $(20 \mathrm{ml})$ and the mixture was stirred for $14 \mathrm{~h}$ at room temperature. After the reaction, the mixture was extracted with ethyl acetate. The organic layer was washed with a saturated aqueous solution of sodium chloride, dried over magnesium sulfate, and condensed in vacuo. The residue was purified by silica gel column chromatography (hexane : diethyl ether $=3: 1)$ to afford $19(382 \mathrm{mg}, 95 \%)$ as colorless needles. mp. $93-94{ }^{\circ} \mathrm{C}$ (hexane/ethyl acetate). ${ }^{1} \mathrm{H}-\mathrm{NMR}\left(400 \mathrm{MHz}, \mathrm{CDCl}_{3}\right) \delta$ : $0.54(3 \mathrm{H}, \mathrm{s}), 1.07(1 \mathrm{H}, \mathrm{dt}, J=13.4,4.0 \mathrm{~Hz}), 1.19(3 \mathrm{H}, \mathrm{s}), 1.23-1.33(1 \mathrm{H}$ $\mathrm{m}), 1.35-1.36(1 \mathrm{H}, \mathrm{m}), 1.42-1.43(1 \mathrm{H}, \mathrm{m}), 1.50-1.56(1 \mathrm{H}, \mathrm{m}), 1.73-$ $1.89(3 \mathrm{H}, \mathrm{m}), 1.93-2.00(3 \mathrm{H}, \mathrm{m}), 2.16-2.21(1 \mathrm{H}, \mathrm{m}), 2.43-2.48(1 \mathrm{H}, \mathrm{m})$, $3.62(3 \mathrm{H}, \mathrm{s}), 3.73-3.88(2 \mathrm{H}, \mathrm{m}), 4.65(1 \mathrm{H}, \mathrm{d}, J=1.1 \mathrm{~Hz}), 4.97(1 \mathrm{H}, \mathrm{dd}$ $J=2.6,1.1 \mathrm{~Hz}) .{ }^{13} \mathrm{C}-\mathrm{NMR}\left(100 \mathrm{MHz}, \mathrm{CDCl}_{3}\right) \delta: 13.5,19.8,26.0,28.8,38.1$, $38.3,39.1,39.5,44.1,51.2,56.0,58.5,58.7,106.3,147.4,177.6$. IR $\left(\mathrm{CHCl}_{3}\right) \mathrm{cm}^{-1}:$ 3692, 3593, 3036, 2993, 2949, 2907, 1717, 1643, 1603, 1468, 1450. EI-MS $m / z: 266.1887$ (Calcd for $\mathrm{C}_{16} \mathrm{H}_{26} \mathrm{O}_{3}:$ 266.1882). MS (70 eV) m/z: $266\left(\mathrm{M}^{+}, 5\right), 248$ (2), 206 (4), 188 (15), 173 (8), 147 (7), 121 (100), 107 (25), $93(21), 81(25), 69(22), 55(36) .[\alpha]_{\mathrm{D}}^{25}=+40.5^{\circ}(c=0.64$, $\left.\mathrm{CHCl}_{3}\right)$.

(-)-Methyl 5-Formylmethyl-6-methylene-1,4a-dimethyldecahydronaphthalene-1-carboxylate (20) Dess-Martin periodinane (167 mg, $0.39 \mathrm{mmol})$ was added to a solution of $19(35 \mathrm{mg}, 0.13 \mathrm{mmol})$ in dichloromethane $(5 \mathrm{ml})$ at $0{ }^{\circ} \mathrm{C}$ and the mixture was stirred for $4 \mathrm{~h}$ at room temperature. After the reaction, the reaction mixture was poured into a saturated aqueous solution of sodium thiosulfate and extracted with diethyl ether. The organic layer was successively washed with a saturated aqueous solution of sodium bicarbonate and sodium chloride, dried over magnesium sulfate and condensed in vacuo. The residue was purified by silica gel column chromatography (hexane: ethyl acetate $=5: 1)$ to afford 20 (34 mg, $98 \%$ ) as colorless needles. mp. $102-104^{\circ} \mathrm{C}$ (hexane/ethyl acetate). ${ }^{1} \mathrm{H}$ NMR (400 MHz, $\left.\mathrm{CDCl}_{3}\right) \delta: 0.97(3 \mathrm{H}, \mathrm{s}), 1.08(1 \mathrm{H}, \mathrm{dt}, J=13.4,4.0 \mathrm{~Hz}), 1.20$ $(3 \mathrm{H}, \mathrm{s}), 1.20-1.30(2 \mathrm{H}, \mathrm{m}), 1.47-1.64(2 \mathrm{H}, \mathrm{m}), 1.80-1.94(2 \mathrm{H}, \mathrm{m})$, $1.97-2.07(2 \mathrm{H}, \mathrm{m}), 2.19-2.24(1 \mathrm{H}, \mathrm{m}), 2.42-2.47(2 \mathrm{H}, \mathrm{m}), 3.66(3 \mathrm{H}, \mathrm{s})$, $4.51(1 \mathrm{H}, \mathrm{m}), 4.94(1 \mathrm{H}, \mathrm{m}), 9.87(1 \mathrm{H}, \mathrm{d}, J=4.9 \mathrm{~Hz}) .{ }^{13} \mathrm{C}-\mathrm{NMR}(100 \mathrm{MHz}$, $\left.\mathrm{CDCl}_{3}\right) \delta: 14.3,19.4,24.8,28.7,37.3,38.0,39.3,40.0,43.9,51.4,55.0$, 67.1, 109.3, 144.6, 177.4, 205.1. IR $\left(\mathrm{CHCl}_{3}\right) \mathrm{cm}^{-1}: 3690,3035,3008.7$, 2993, 2910, 1717, 1645, 1602, 1466, 1450, 1441. EI-MS m/z: 264.1725 (Calcd for $\mathrm{C}_{16} \mathrm{H}_{24} \mathrm{O}_{3}:$ 264.1732). MS (70 eV) m/z: $264\left(\mathrm{M}^{+}, 25\right), 249$ (5), 232 (9), 220 (8), 204 (37), 189 (19), 181 (10), 161 (16), 149 (20)135 (17) 121 (100), 109 (30), $95(25), 81(22), 67(15), 55(21) .[\alpha]_{\mathrm{D}}^{25}=-34.3^{\circ}$ $\left(c=0.50, \mathrm{CHCl}_{3}\right)$. Anal. Calcd for $\mathrm{C}_{16} \mathrm{H}_{24} \mathrm{O}_{3}: \mathrm{C}, 72.69 ; \mathrm{H}, 9.15$. Found: $\mathrm{C}$, $72.72 ; \mathrm{H}, 9.03$.

(-)-Methyl 13-Oxo-15,16-dinorlabda-8(17),11E-dien-19-oate (4) 1Triphenylphosphoranylidene-2-propanone $(63 \mathrm{mg}, 0.20 \mathrm{mmol})$ was added to a solution of $20(17 \mathrm{mg}, 0.065 \mathrm{mmol})$ in toluene $(2 \mathrm{ml})$ and the mixture was stirred for $24 \mathrm{~h}$ at refluxing temperature. After the reaction, the organic solvent was removed in vacuo and the residue was purified by silica gel column chromatography (hexane : ethyl acetate $=5: 1)$ to afford $4(9 \mathrm{mg}, 45 \%)$ as a colorless oil. ${ }^{1} \mathrm{H}-\mathrm{NMR}\left(400 \mathrm{MHz}, \mathrm{CDCl}_{3}\right) \delta: 0.71(3 \mathrm{H}, \mathrm{s}), 1.02-1.14(2 \mathrm{H}$, $\mathrm{m}), 1.21(3 \mathrm{H}, \mathrm{s}), 1.32(1 \mathrm{H}, \mathrm{dd}, J=12.5,2.8 \mathrm{~Hz}), 1.42-1.50(2 \mathrm{H}, \mathrm{m}), 1.73$ $1.89(2 \mathrm{H}, \mathrm{m}), 1.97-2.07(2 \mathrm{H}, \mathrm{m}), 2.17-2.23(1 \mathrm{H}, \mathrm{m}), 2.27(3 \mathrm{H}, \mathrm{s}), 2.43$ $2.49(2 \mathrm{H}, \mathrm{m}), 3.65(3 \mathrm{H}, \mathrm{s}), 4.42(1 \mathrm{H}, \mathrm{dd}, J=3.0,1.5 \mathrm{~Hz}), 4.81(1 \mathrm{H}, \mathrm{dd}$ $J=3.0,1.5 \mathrm{~Hz}), 6.07(1 \mathrm{H}, \mathrm{d}, J=15.9 \mathrm{~Hz}), 6.85(1 \mathrm{H}, \mathrm{dd}, J=15.9,10.3 \mathrm{~Hz})$ ${ }^{13} \mathrm{C}-\mathrm{NMR}\left(100 \mathrm{MHz}, \mathrm{CDCl}_{3}\right) \delta: 13.5,19.6,25.0,27.3,28.7,37.1,38.2$, $39.7,40.9,44.2,51.3,55.4,60.0,108.3,133.7,146.0,148.1,177.5,198.0$ IR $\left(\mathrm{CHCl}_{3}\right) \mathrm{cm}^{-1}: 3690,3038,3001,1719,1670,1645,1624,1603,1545$ 1533, 1466, 1434. EI-MS $m / z: 304.2038$ (Calcd for $\mathrm{C}_{19} \mathrm{H}_{28} \mathrm{O}_{3}: 304.2043$ ). MS (70 eV) m/z: $304\left(\mathrm{M}^{+}, 4\right), 289$ (2), 261 (3), 244 (6), 201 (10), 161 (13), 137 (31), 121 (100), 109 (52), 93 (39), 81 (99), 77 (28), 55 (53). $[\alpha]_{\mathrm{D}}^{25}=-1.61^{\circ}\left(c=0.16, \mathrm{CHCl}_{3}\right)$

Methyl 13-Hydroxy-15,16-dinorlabda-8(17),11E-dien-19-oate (3) Sodium borohydride $(2 \mathrm{mg}, 0.056 \mathrm{mmol})$ was added to a solution of cerium chloride $(14 \mathrm{mg}, 0.056 \mathrm{mmol})$ in methanol $(1 \mathrm{ml})$, to which $4(6 \mathrm{mg}$, $0.018 \mathrm{mmol}$ ) was added and the mixture was stirred for $1 \mathrm{~h}$ at $0^{\circ} \mathrm{C}$. After the reaction, the mixture was poured into a saturated aqueous solution of ammonium chloride and extracted with ethyl acetate. The organic layer was washed with a saturated aqueous solution of sodium chloride, dried over magnesium sulfate, and condensed in vacuo. The residue was purified by silica gel column chromatography (hexane : ethyl acetate $=3: 1$ ) to afford a mixture of diastereomers of $\mathbf{3}(6 \mathrm{mg}, 99 \%)$ as colorless amorphous. ${ }^{1} \mathrm{H}$ -
NMR $\left(400 \mathrm{MHz}, \mathrm{CDCl}_{3}\right) \delta: 0.60(1.5 \mathrm{H}, \mathrm{s}), 0.62(1.5 \mathrm{H}, \mathrm{s}), 1.01-1.09(2 \mathrm{H}$ m), $1.20(3 \mathrm{H}, \mathrm{s}), 1.27(1.5 \mathrm{H}, \mathrm{d}, J=6.4 \mathrm{~Hz}), 1.28(1.5 \mathrm{H}, \mathrm{d}, J=6.4 \mathrm{~Hz}), 1.31-$ $1.32(1 \mathrm{H}, \mathrm{m}), 1.44-1.58(3 \mathrm{H}, \mathrm{m}), 1.74-1.85(2 \mathrm{H}, \mathrm{m}), 1.93-2.04(2 \mathrm{H}, \mathrm{m})$, $2.14-2.19(1 \mathrm{H}, \mathrm{m}), 2.28-2.30(1 \mathrm{H}, \mathrm{m}), 2.41-2.46(1 \mathrm{H}, \mathrm{m}), 3.63(1.5 \mathrm{H}$, s), $3.63(1.5 \mathrm{H}, \mathrm{s}), 4.33(1 \mathrm{H}$, quint., $J=6.4 \mathrm{~Hz}), 4.46(0.5 \mathrm{H}, \mathrm{d}, J=1.7 \mathrm{~Hz})$, $4.51(0.5 \mathrm{H}, \mathrm{d}, J=1.1 \mathrm{~Hz}), 4.75-4.77(1 \mathrm{H}, \mathrm{m}), 5.51(0.5 \mathrm{H}, \mathrm{dd}, J=15.4$, $6.5 \mathrm{~Hz}), 5.52(0.5 \mathrm{H}, \mathrm{dd}, J=15.4,6.5 \mathrm{~Hz}), 5.68(1 \mathrm{H}, \mathrm{dd}, J=15.4,9.7 \mathrm{~Hz}) . \mathrm{IR}$ $\left(\mathrm{CHCl}_{3}\right) \mathrm{cm}^{-1}: 2947,2850,1717,1450,1381,1246,1157$. EI-MS $\mathrm{m} / z$ : 306.2196 (Calcd for $\left.\mathrm{C}_{19} \mathrm{H}_{30} \mathrm{O}_{3}: 306.2195\right)$. MS $(70 \mathrm{eV}) \mathrm{m} / \mathrm{z}: 306\left(\mathrm{M}^{+}, 1\right)$, 288 (6), 246 (8), 213(9), 188 (13), 159 (15), 121 (100), 105 (23), 93 (32), 79 (22), 67 (15), 55 (20).

13-Hydroxy-15,16-dinorlabda-8(17),11E-dien-19-oic Acid (22) $n$ Butyllithium ( $2.46 \mathrm{M}$ solution in $n$-hexane, $104 \mu \mathrm{l}, 0.28 \mathrm{mmol}$ ) was added to a solution of 1-dodecanethiol $(66 \mu 1,0.28 \mathrm{mmol})$ in hexamethylphosphoramide and the mixture was stirred for $30 \mathrm{~min}$ at room temperature. The allyl alcohol 3 ( $9 \mathrm{mg}, 0.028 \mathrm{mmol}$ ) was added to the reaction mixture, which was stirred for $30 \mathrm{~min}$. After the reaction, the mixture was poured into a saturated aqueous solution of ammonium chloride and extracted with diethyl ether. The organic layer was washed with distilled water, dried over magnesium sulfate, and condensed in vacuo. The residue was purified by silica gel column chromatography (hexane : ethyl acetate $=4: 1$ ) to afford a mixture of diastereomers of 22 (8 mg, quantitative) as colorless amorphous. ${ }^{1} \mathrm{H}-\mathrm{NMR}$ $\left(400 \mathrm{MHz}, \mathrm{CDCl}_{3}\right) \delta: 0.70(1.5 \mathrm{H}, \mathrm{s}), 0.71(1.5 \mathrm{H}, \mathrm{s}), 1.02-1.10(2 \mathrm{H}, \mathrm{m})$, $1.24-1.35(2 \mathrm{H}, \mathrm{m}), 1.26(3 \mathrm{H}, \mathrm{s}), 1.28(1.5 \mathrm{H}, \mathrm{d}, J=6.5 \mathrm{~Hz}), 1.28(1.5 \mathrm{H}, \mathrm{d}$ $J=6.5 \mathrm{~Hz}), 1.45-1.59(2 \mathrm{H}, \mathrm{m}), 1.76-2.04(4 \mathrm{H}, \mathrm{m}), 2.15-2.19(1 \mathrm{H}, \mathrm{m})$, $2.29-2.31(1 \mathrm{H}, \mathrm{m}), 2.43-2.47(1 \mathrm{H}, \mathrm{m}), 4.34(0.5 \mathrm{H}, \mathrm{t}$ of quint., $J=6.4$, $1.1 \mathrm{~Hz}), 4.46(0.5 \mathrm{H}, \mathrm{d}, J=1.1 \mathrm{~Hz}), 4.51(0.5 \mathrm{H}, \mathrm{d}, J=1.1 \mathrm{~Hz}), 4.75-4.77$ $(1 \mathrm{H}, \mathrm{m}), 5.52(0.5 \mathrm{H}, \mathrm{dd}, J=15.4,6.5 \mathrm{~Hz}), 5.52(0.5 \mathrm{H}, \mathrm{dd}, J=15.4,6.5 \mathrm{~Hz})$, $5.68(1 \mathrm{H}, \mathrm{dd}, J=15.4,9.7 \mathrm{~Hz})$. IR $\left(\mathrm{CHCl}_{3}\right) \mathrm{cm}^{-1}: 3028,2936,2851,1693$, 1230. FAB-MS $m / z$ : 315.1942 (Calcd for $\mathrm{C}_{18} \mathrm{H}_{28} \mathrm{O}_{3} \mathrm{Na}: 315.1936$ ). MS (FAB) $m / z: 315\left(\mathrm{M}+\mathrm{Na}^{+}, 100\right)$.

(-)-13-Oxo-15,16-dinorlabda-8(17),11E-dien-19-oic Acid (1) Pyridinium dichromate $(26 \mathrm{mg}, 0.07 \mathrm{mmol})$ and Celite ${ }^{\mathbb{R}}(26 \mathrm{mg})$ were added to a solution of $22(8 \mathrm{mg}, \mathrm{mmol})$ in $N, N$-dimethylformamide $(1 \mathrm{ml})$ and the mixture was stirred for $7 \mathrm{~h}$ at room temperature. After the reaction, the organic solvent was removed in vacuo and purified by silica gel column chromatography (hexane : ethyl acetate $=3: 1)$ to afford $1(8 \mathrm{mg}, 99 \%)$ as colorless crystals. The ${ }^{1} \mathrm{H}-\mathrm{NMR}$ spectrum of our synthesized $\mathbf{1}$ coincided with that of naturally occurring $\mathbf{1}^{6}{ }^{6}$

Acknowledgements This research was financially supported in part by Frontier Research Program and the 21st Century Center of Excellence Program "Development of Drug Discovery Frontier Integrated from Tradition to Proteome" of the Ministry of Education, Culture, Sport, Science and Technology of Japan.

\section{References}

1) Tanaka R., Ohtsu H., Iwamoto M., Minami T., Tokuda H., Nishino H., Matsunaga S., Yoshitake A., Cancer Lett., 161, 165-170 (2000).

2) Narisawa T., Fukaura Y., Hasebe M., Noura S., Ohshima S., Sakamoto H., Inakuma T., Takayasu J., Nishino H., J. Cancer Res., 89, 10031008 (1988).

3) Inoue M., Hasegawa S., Hirose Y., Phytochemistry, 24, 1602-1604 (1985).

4) Fang J.-M., Chen W.-C., Wang B.-W., Cheng Y.-S., Phytochemistry, 41, 1361-1365 (1996)

5) Kuo Y.-H., Cheng W.-C., J. Chin. Chem. Soc., 46, 819-824 (1999).

6) Katoh T., Tanaka R., Takeo M., Nishide K., Node M., Chem. Pharm. Bull., 50, 1625-1629 (2002).

7) Katoh T., Mizumoto S., Fudesaka M,, Takeo M., Kajimoto T., Node M., Tetrahedron: Asymmetry, 17,1655-1662 (2006).

8) Scott W., Sward P. K., Stoessel S. J., Stille J. K., J. Org. Chem., 50, 2302-2308 (1985).

9) Umbreit M. A., Sharpless K. B., J. Am. Chem. Soc., 99, 5526-5528 (1977).

10) Toshima H., Okikawa H., Toyomatsu T., Sassa T., Tetrahedron, 56, 8443 - 8450 (2000).

11) Gemal A., Luche J.-L., J. Am. Chem. Soc., 103, 5454-5459 (1981).

12) Node M., Kumar K., Nishide K., Ohsugi S., Miyamoto T., Tetrahedron Lett., 42, 9207-9210 (2001).

13) Nishide K., Miyamoto T., Kumar K., Ohsugi S., Node M., Tetrahedron Lett., 43, 8569-8573 (2002). 